\title{
Treatment after failure: the problem of "non-responders"
}

\author{
J-Q Huang, R H Hunt
}

\begin{abstract}
Summary
Although the currently most effective treatment regimens cure about $90 \%$ of infections, $10 \%$ of patients remain Helicobacter pylori positive. Several factors contribute to treatment failure. These include patient compliance, bacterial resistance to antibiotics, and treatment related issues. Treatment failure leads to the development of bacterial resistance to metronidazole and clarithromycin. Retreatment can be undertaken after considering several different strategies: to repeat the same regimen with full doses of medications and a longer treatment duration, or to choose different regimens to avoid the antibiotic previously used, or to switch to proton pump inhibitor (PPI) based quadruple therapy or ranitidine bismuth citrate (RBC) based triple therapy. In principle, full doses and longer treatment durations are advisable. As retreatment is always difficult, choosing the best available first line treatment regimen is still the best "rescue" treatment.
\end{abstract}

\section{Introduction}

It has been over 12 years since the first randomised, placebo controlled clinical trial for the eradication of $H$ pylori infection was published. ${ }^{1}$ Treatment to eradicate the infection has evolved from single agents to multiple combination treatments consisting of an antisecretory agent and one or more antibiotics. ${ }^{2} 3$ Treatments that achieve an eradication rate of greater than $80 \%$, on an intent to treat basis, have been recommended by most consensus conferences and authorities. ${ }^{4-7}$ These now include bismuth based triple therapy, triple therapy involving a PPI and two antibiotics, quadruple therapy, or more recently combinations with RBC and two antibiotics. Although these combination treatments are very effective for eradicating $H$ pylori infection in most patients, a significant proportion of patients fail these treatments for a variety of different reasons such as bacterial resistance, poor compliance, or treatment related factors. ${ }^{89}$ Retreatment of these non-responders remains a challenging issue as some patients may need

ndence to: Professor Hunt.

Table 1 H pylori eradication: pooled success and failure rates with the most commonly used regimens

\begin{tabular}{|c|c|c|c|c|c|c|}
\hline \multirow[b]{2}{*}{ Regimen * } & \multicolumn{3}{|c|}{ Success (ITT) } & \multicolumn{3}{|c|}{ Failure (\%) } \\
\hline & $n$ & $\%$ & $95 \% C I$ & Mean & Range & Reference \\
\hline PAC500 & 514 & 90 & $86-94$ & 10 & $6-14$ & Huang and colleagues ${ }^{10}$ \\
\hline PMC500 & 253 & 91 & $81-100$ & 9 & $0-19$ & Huang and colleagues ${ }^{10}$ \\
\hline PBMT & 611 & 90 & $86-94$ & 10 & $6-14$ & Huang and colleagues ${ }^{10}$ \\
\hline RCA & 521 & 82 & $79-85$ & 18 & $15-21$ & Pipkin and colleagues ${ }^{11}$ \\
\hline RMC & 791 & 89 & $87-91$ & 11 & $9-13$ & Pipkin and colleagues ${ }^{11}$ \\
\hline
\end{tabular}

*See text for definitions.

$\mathrm{CI}$, confidence interval; ITT, intent to treat; $\mathrm{n}$, total number of patients. more than two attempts for eradication of the infection. This paper reviews the current literature on the possible causes for $H$ pylori treatment failure, discusses several approaches to retreatment, and gives suggestions for future studies.

\section{How serious is the problem of treatment failure?}

To answer this question, we need to assess the efficacy of the currently most recommended treatment regimens. In a comprehensive metaanalysis involving 294 treatment arms and 15971 patients, we found that the most effective treatment regimens reported up to 1997 were seven day triple therapy consisting of a PPI, clarithromycin (C) $500 \mathrm{mg}$ twice daily, and amoxcillin (A) or metronidazole (M), or quaduple therapy with a PPI and bismuth (B), metronidazole $(\mathrm{M})$, and tetracycline $(\mathrm{T}){ }^{10}$ These regimens achieve a pooled eradication rate of at least $90 \%$ by intent to treat analysis. However, a large variation in efficacy exists between studies in these treatment regimens, with $95 \%$ confidence intervals (CI) ranging from $81 \%$ to $100 \%$. This means that up to $19 \%$ of patients will fail these treatments and remain $H$ pylori positive (table 1 ). In a recent review of RBC based triple therapy, Pipkin et al showed that the pooled eradication rate for seven day RBC, clarithromycin, and metronidazole was $89 \%(95 \%$ CI 87 to $91 \%)$ by intent to treat analysis and $82 \%$ (95\% CI 79 to $85 \%$ ) for RBC, clarithromycin, and amoxicillin, respectively. ${ }^{11}$ This suggests that up to $21 \%$ of patients will fail RBC based triple therapy and require retreatment for the infection.

\section{Factors leading to treatment failure} PATIENT RELATED FACTORS

Poor compliance

Poor compliance has been considered a major factor for treatment failure. In a stepwise regression analysis of factors influencing the effectiveness of bismuth based triple therapy, Graham et al have shown that patient compliance was the most important factor predicting treatment success. ${ }^{9}$ The $H$ pylori cure rate was $96 \%$ for patients who took more than $60 \%$ of the prescribed medications and only $69 \%$ for those who took less $(\mathrm{p}=0.001)$. In a recent study of treatment failure of $H$ pylori infection in an ambulatory population, $30 \mathrm{H}$ pylori infected patients were treated with lansoprazole (L) $30 \mathrm{mg}$ twice daily, amoxicillin $1000 \mathrm{mg}$ twice daily, and clarithromycin $500 \mathrm{mg}$ twice

Abbreviations used in this paper: PPI, proton pump inhibitor; RBC, ranitidine bismuth citrate; PUD, peptic ulcer disease; NUD, non-ulcer dyspepsia. 
daily for seven days. ${ }^{12}$ Patient compliance was assessed by the Medication Event Monitoring System (MEMS) containers, which contained a concealed electronic device recording the time of opening. The overall eradication rate was $63 \%$. In patients who took more than $60 \%$ of the stipulated medications, the cure rate was $72 \%(18 / 25)$, whereas the eradication rate was only $20 \%(1 / 5)$ in those who took less than $60 \%$ of the prescribed pills $(p=0.028) .{ }^{12}$ These results confirm that patient compliance plays an important role in the success of $H$ pylori eradication treatment. However, a significant proportion of the treatment failures in this study cannot be explained by the poor compliance as the eradication rate was only $72 \%$ in patients who had taken more than $60 \%$ of the prescribed medications.

\section{Bacterial resistance}

Bacterial resistance to metronidazole or clarithromycin is an important factor leading to treatment failure. There is a good correlation between bacterial resistance to clarithromycin and eradication failure; however, the clinical relevance of $H$ pylori resistance to nitroimidazoles detected in vitro has been controversial. Some studies suggest that triple therapy consisting of a PPI, clarithromycin, and a nitroimidazole are effective at eradicating $H$ pylori infection in patients harbouring metronidazole resistant strains ${ }^{13-15}$; others show that the efficacy is significantly decreased when metronidazole resistance is present. ${ }^{16-19}$ Several factors might have contributed to these differences, including different methods used for diagnosing metronidazole resistance, ${ }^{20}{ }^{21}$ different cut off values for determining the minimum inhibitory concentrations, ${ }^{22} 23$ and probably different locations where biopsy samples were taken. ${ }^{24}$ In patients who harbour metronidazole resistant strains, the eradication rate is about $30 \%$ lower than in those infected with metronidazole sensitive strains, when treated with bismuth, metronidazole, and tetracycline or amoxicillin triple therapy. ${ }^{8}$ Although a PPI can overcome partially the impact of metronidazole resistance on $H$ pylori eradication, ${ }^{25}$ the eradication rate achieved with PAM in patients infected with metronidazole resistant strains is about $30 \%$ lower than in those who are infected with metronidazole sensitive strains. ${ }^{8232627}$ A similar difference is also seen when patients are treated with the PMC combination. ${ }^{8} 28$

The prevalence of $H$ pylori resistance to clarithromycin is generally much lower than that to metronidazole worldwide. ${ }^{8}$ However, the resistance of $H$ pylori to clarithromycin has a significant impact on treatment success for regimens containing this drug. The presence of primary bacterial resistance to clarithromycin is almost $100 \%$ predictive of treatment failure with dual therapy consisting of omeprazole and clarithromycin. ${ }^{29}$ Hence dual therapy should not be used. In studies with PAC combinations given for 10-14 days, $H$ pylori eradication rates ranged from $83 \%$ to $98 \%$ in patients infected with clarithromycin sensitive strains, whereas only $25-50 \%$ of infections were eradicated in those who harboured strains resistant to clarithromycin. $^{8}$

The secondary bacterial resistance to metronidazole or clarithromycin increases significantly in patients who have failed previous eradication treatments. Buckley et al reported that, of 87 patients treated with seven day PMC (omeprazole $20 \mathrm{mg}$ was given once daily), 31 patients $(35.6 \%)$ were infected with metronidazole resistant strains and three $(3.4 \%)$ were infected with strains resistant to both antibiotics. ${ }^{16}$ Secondary bacterial resistance to clarithromycin developed in seven $(58.3 \%)$ of the 12 patients with metronidazole resistant strains who failed treatment. ${ }^{16}$ In another study, metronidazole resistance was detected in $28 \%$ of patients $(7 / 25)$ before eradication treatment but increased to $66.7 \%(12 / 18)$ after unsuccessful treatment with metronidazole containing regimens. ${ }^{30}$ Clarithromycin resistant strains also increased from $32 \%$ before treatment to $70.6 \%$ after unsuccessful treatment. ${ }^{30}$ This suggests that previous treatment failure is a significant contributing factor to acquired bacterial resistance to both metronidazole and clarithromycin.

\section{$H$ pylori strains}

The results from recent studies also indicate that the cag $\mathrm{A}^{-}$status of bacterial strains is also a risk factor for treatment failure. In a multicentre study, Marais et al have shown that $H$ pylori eradication was achieved in $87 \%$ of patients (54/62) infected with cagA ${ }^{+}$strains, when treated with seven day PPI based triple therapy compared with $69 \%(47 / 68)$ of those harbouring cagA $\mathrm{A}^{-}$strains. $^{31}$ This observation may explain, at least in part, the difference in $H$ pylori eradication rates, which are reported between patients with peptic ulcer disease (PUD) and those with non-ulcer dyspepsia (NUD), when patients are treated with the same regimens. ${ }^{32}$ Virulent strains of $H$ pylori infection are more prevalent in patients with PUD than in those with NUD. ${ }^{33}$ This might have contributed to the higher eradication rates achieved in patients with PUD compared with NUD in some studies. ${ }^{34} 35$ However, as discussed before, patient compliance plays an important role in determining the success of treatment. In comparison with patients with PUD, those with NUD may tolerate eradication treatment less well due to the refractoriness and diverse nature of symptoms. Indeed, when patient compliance is greater than $90 \%$, eradication can be achieved in over $90 \%$ of NUD patients treated with a PPI, clarithromycin, and amoxicillin or nitroimidazole. ${ }^{3637}$

\section{TREATMENT RELATED FACTORS}

Components of a regimen

The components of a treatment regimen also play an important role in $H$ pylori eradication, such as which drugs are selected, the number and doses of medications used in a combination, dosing frequency, and treatment duration. PPI based triple therapy is significantly more effective for $H$ pylori eradication than dual therapy consisting of two antibiotics or a PPI plus an antibiotic. ${ }^{28}$ PPI given twice daily is 
significantly more effective than once daily in combination with clarithromycin and amoxicillin or nitroimidazole. ${ }^{11} 38$ PPI based triple therapy may eradicate more $H$ pylori infections when given for 10-14 days than for seven days. ${ }^{39}$ Therefore, a good combination therapy should include full doses of medications, optimal dosing frequency, and adequate treatment duration.

\section{Strategies for rescue treatment after failure}

Several steps can be taken in the management of patients with treatment failure. These include the determination of the status of bacterial resistance to metronidazole, and/or clarithromycin (or less likely to amoxicillin), and patient compliance with the previous treatment regimen. This information is helpful for selecting a proper rescue therapy as to whether retreatment should be given with full doses of the medications and for longer with the same combination, or with different combinations to avoid the antibiotic previously used when bacterial resistance to the antibiotic is suspected.

Ricci et al reported a study of $123 \mathrm{H}$ pylori infected patients who were treated with a PPI $20 \mathrm{mg}$ twice daily, amoxicillin $1 \mathrm{~g}$ twice daily, and clarithromycin $250 \mathrm{mg}$ twice daily for seven days. ${ }^{40} \mathrm{H}$ pylori eradication was achieved in $85 \%$ of the 123 patients. Fifteen patients failed the therapy and remained $H$ pylori positive four weeks after treatment. These patients underwent a second course of eradication treatment with full dose clarithromycin $500 \mathrm{mg}$ twice daily and 10 days' treatment. $H$ pylori infection was cured in $67 \%(6 / 9)$ of the evaluable patients. ${ }^{40}$ This result suggests that retreatment of $H$ pylori infection with the same combination is still a choice when the status of bacterial resistance to antibiotics is not known. However, full doses and a longer treatment duration must be used. If patients are treated with the same combination and for the same treatment duration, a poor eradication rate is usually reported. ${ }^{41}$

As acquired bacterial resistance to metronidazole or clarithromycin results primarily from the previous treatment failure, rescue therapies generally should avoid these antibiotics and use different combinations in order to achieve a high eradication rate. PPI based triple therapy is generally very effective for treating patients who have failed PPI based dual therapy. ${ }^{42}$ In the event of PPI based triple therapy failure such as seven days' omeprazole $(\mathrm{O})$, amoxicillin $(\mathrm{A})$, and metronidazole $(\mathrm{M})$, retreatment with seven days' omeprazole $20 \mathrm{mg}$ twice daily, amoxicillin $1 \mathrm{~g}$ twice daily, and clarithromycin (C) $500 \mathrm{mg}$ twice daily has proved to be very effective with a success rate of $85 \% .{ }^{44}$ When metronidazole and/or clarithromycin resistance is present, retreatment with seven days OMC has been shown, in a randomised controlled study by Houben et al, ${ }^{41}$ to be ineffective in salvaging therapeutic failure, when compared to seven days' quadruple therapy consisting of bismuth (B), metronidazole (M), tetracycline (T), and omeprazole. $H$ pylori eradication was achieved in $100 \%(11 / 11)$ of the patients treated with OBMT, including eight patients infected with metronidazole resistant and/or clarithromycin resistant $H$ pylori strains, whereas the success rate was only $25 \%(2 / 8)$ in patients given OMC. None of the patients who was infected with metronidazole resistant and/or clarithromycin resistant strains responded to OMC. ${ }^{41}$

More recent data suggest that RBC based triple therapy may overcome the impact of metronidazole resistant and clarithromycin resistant strains on $H$ pylori eradication treatment. In a study reported by Wouden et al, 111 patients infected with $H$ pylori were treated with RBC $400 \mathrm{mg}$ twice daily, clarithromycin $500 \mathrm{mg}$ twice daily, and metronidazole $500 \mathrm{mg}$ twice daily for seven days. The overall success rate was $96 \%$ by intent to treat analysis. The infection was cured in $95 \%(20 / 21)$ of the patients harbouring metronidazole resistant strains, and in $100 \%(4 / 4)$ of those who were infected with clarithromycin resistant strains or with metronidazole resistant and clarithromycin resistant strains. ${ }^{45}$ As the number of patients included in this study was small, these promising results need to be confirmed by future studies. One recent anecdotal report has also suggested that furazolidone can be used to replace either clarithromycin or metronidazole in PPI based triple therapy for $H$ pylori treatment. ${ }^{46}$ In this small study of 12 patients infected with metronidazole sensitive and clarithromycin sensitive strains of $H$ pylori, Graham et al reported that triple therapy, consisting of furazolidone $100 \mathrm{mg}$ three times daily, omeprazole $20 \mathrm{mg}$ four times daily, and metronidazole $500 \mathrm{mg}$ three times daily or clarithromycin $500 \mathrm{mg}$ three times daily given for 14 days, successfully eradicated all infections. ${ }^{46}$ This promising result also needs to be confirmed in larger, randomised studies.

\section{Recommendations from major consensus conferences}

Recommendations for retreatment of $H$ pylori infection have been made in several major consensus conferences worldwide. ${ }^{4-7}$ The European Helicobacter pylori Study Group recommended that, in the case of treatment failure, a retreatment regimen should be selected after consideration of previous treatment or microbial sensitivities, or both. PPI based quadruple therapy can be used in the event of failure of triple therapy. ${ }^{6}$ In the Asian-Pacific Consensus, it is suggested that, following treatment failure with PCA or RBC based clarithromycin and amoxicillin combination, the same regimen may be repeated. ${ }^{7}$ Following one treatment failure with a regimen containing metronidazole, treatment may be repeated, substituting amoxicillin for metronidazole. Seven days PPI based quadruple therapy was also recommended as a back up treatment by the panel. ${ }^{7}$ In the United States, treatment duration is longer than that recommended in Europe, the Asia-Pacific region, and Canada. ${ }^{4}$ Two weeks' treatment is recommended by the US consensus meeting. ${ }^{4}$ The extended treatment duration is important in the management of treatment 
failure, which can increase the success rate of retreatment. ${ }^{40}$

\section{What is the best "rescue" strategy?}

As previous treatment failure leads to the development of secondary bacterial resistance to both metronidazole and clarithromycin ${ }^{30}$ and the efficacy of retreatment is also significantly decreased by the number of previous treatments, ${ }^{47}$ it is understandable that the higher success rate with the first line treatment, the lower will be the treatment failure. Thus, choosing the best available first line treatment regimen should be considered to be the best approach to "rescue" treatment. In areas where the prevalence of metronidazole resistance in the general population is high, seven days' triple therapy including a PPI at the recommended doses, clarithromycin $500 \mathrm{mg}$ twice daily, and amoxicillin $1 \mathrm{~g}$ twice daily should be used if the bacterial sensitivity is not known. In the event of clarithromycin resistance, a seven day PPI based quadruple therapy or 14 day $\mathrm{H}_{2}$ receptor antagonist based quadruple therapy should be considered. ${ }^{5}$ The PPI and $\mathrm{H}_{2}$ receptor antagonist should be given at the recommended doses. Colloidal bismuth salicylate is given as $120 \mathrm{mg}$ four times daily, metronidazole as $250 \mathrm{mg}$ four times daily, and tetracycline as $500 \mathrm{mg}$ four times daily. ${ }^{5}$ RBC $400 \mathrm{mg}$ twice daily given with clarithromycin $500 \mathrm{mg}$ twice daily and amoxicillin $1 \mathrm{~g}$ twice daily for seven days may also be considered as an alternative first line treatment regimen.

\section{Conclusion}

In conclusion, the information on treatment failure of $H$ pylori infection in the current literature is scarce. Many reports are published only in abstract form and are limited by the small number of patients and study design. However, even with the current most effective treatment regimens, about $10 \%$ of patients will fail a variety of treatments and remain $H$ pylori positive. Patient compliance, bacterial resistance to antibiotics, and the components of the regimen should be assessed when retreatment is considered. Full doses of all components of the combination should be used and treatment given for two weeks. As acquired bacterial resistance mainly results from previous treatment failure, the most effective first line treatment regimen should be regarded as the best "rescue" treatment strategy.

1 McNulty CAM, Gearty JC, Crump B, et al. Campylobacter pyloridis and associated gastritis: investigator blind, placebo controlled trial of bismuth salicylate and erythromycin ethylsuccinate. BMF 1986;293:645-9.

2 Huang JQ, Hunt RH. Review: eradication of Helicobacter pylori: problems and recommendations. $\mathcal{F}$ Gastroentero Hepatol 1997;12:590-8.

3 Hunt RH. Eradication of Helicobacter pylori infection. Am f Med 1996;100(suppl 5A):42-51S.

4 Peura DA and the American Digestive Health Foundation. The report of the Digestive Health Initiative international update conference on Helicobacter pylori. Gastroenterology 1997;113:S4-8.

5 Hunt RH, Thomson ABR. Canadian Helicobacter pylori consensus conference. Can f Gastroenterol 1998;12:31-41.

6 European Helicobacter Pylori Study Group. Current European concepts in the management of Helicobacter pylori infection. The Maastricht Consensus Report. Gut 1997;41: $8-13$.
7 Lam SK, Talley NJ. Helicobacter pylori consensus: report of the 1997 Asia Pacific consensus conference on the management of Helicobacter pylori infection. 7 Gastroenterol Hepatol 1998;13:1-12.

8 Glupczynski Y. Antimicrobial resistance in Helicobacter pylori: a global overview. In: Hunt RH, Tytgat GNJ, eds. Helicobacter pylori: basic mechanisms to clinical cure 1998 London: Kluwer Academic Publishers, 1998:398-415.

9 Graham D, Lew GM, Malaty HM, et al. Factors influencing the eradication of Helicobacter pylori with triple therapy. Gastroenterology 1992;102:493-6.

10 Huang J-Q, Chiba N, Wilkinson JM, et al. Which combination therapy can eradicate $\geqslant 90 \%$ Helicobacter pylori $(\mathrm{H}$. pylori) infection? A meta-analysis of amoxicillin, metronidazole, tetracycline and clarithromycin containing regimens [abstract]. Gastroenterology 1997;112:A19.

11 Pipkin GA, Williamson R, Wood JR. Review article: one-week clarithromycin triple therapy regimens for eradication of Helicobacter pylori. Aliment Pharmacol Ther 1998;12:823-37.

12 Wermeille J, Dederding JP, Cunningham M, et al. Failure of Helicobacter pylori eradication in an ambulatory population: is poor compliance the main cause? Digestion 1998;59(suppl 3):437.

13 Goh KL, Parasakthi N, Chuah SY, et al. Comparison of two 1-week low-dose omeprazole triple therapies-optimal treatment for Helicobacter pylori infection? Aliment Pharmacol Ther 1997;11:1115-18.

14 Adamek RJ, Suerbaum S, Pfaffenbach B, et al. Primary and acquired Helicobacter pylori resistance to clarithromycin, metronidazole, and amoxicillin-influence on treatment outcome. Am f Gastroenterol 1998;93:386-9.

15 Bazzoli F, Zagari M, Pozzato P, et al. Evaluation of short-term low-dose triple therapy for the eradication of Helicobacter pylori by factorial design in a randomized, double-blind, controlled study. Aliment Pharmacol Ther 1998;12:439-45.

16 Buckley MJ, Xia HX, Hyde DM, et al. Metronidazole resistance reduces efficacy of triple therapy and leads to secondary clarithromycin resistance. Dig Dis Sci 1997;42:211115.

17 Harris AW, Pryce DI, Gabe SM, et al. Lansoprazole, clarithromycin and metronidazole for seven days in Helicobacter pylori infection. Aliment Pharmacol Ther 1996;10: $1005-8$

18 Misiewicz JJ, Harris AW, Bardhan KD, et al. One week triple therapy for Helicobacter pylori: a multicentre comparative study. Lansoprazole Helicobacter Study Group. Gut 1997; 41:735-9.

19 Peitz U, Nusch A, Tillenburg B, et al. High cure rate of $\mathrm{H}$. pylori (HP) infection by one-week therapy with omeprazole (OME), metronidazole (MET) and clarithromycin (CLA) despite a negative impact by MET resistance [abstract]. Gut 1996;39(suppl 2):A5

20 Moayyedi P, Ragunathan PL, Mapstone N, et al. Relevance of antibiotic sensitivities in predicting failure of omeprazole, clarithromycin, and tinidazole to eradicate Helicobacter pylori. $f$ Gastroenterol 1998;33(suppl 10):62-5.

21 Hachem CY, Clarridge JE, Reddy R, et al. Antimicrobial susceptibility testing of Helicobacter pylori. Comparison of E-test, broth microdilution, and disk diffusion for ampicillin, clarithromycin, and metronidazole. Diagn Microbiol Infect Dis 1996;24:37-41

22 Xia H, Keane CT, Beattie S, et al. Standardization of disk diffusion test and its clinical significance for susceptibility testing of metronidazole against Helicobacter pylori. testing of metronidazole against Helicobact

23 Megraud F, Doermann HP. Clinical relevance of resistant strains of Helicobacter pylori: a review of current data. Gut 1998;43(suppl 1):S61-5.

24 Xia HH, Kalantar J, Talley NJ. Metronidazole- and clarithromycin-resistant Helicobacter pylori in dyspeptic patients in western Sydney as determined by testing multiple isolates from different gastric sites. 7 Gastroenterol Hepatol 1998;13:1044-9.

25 Lind T, Mégraud F, Bardhan KD, et al. The MACH 2 study: antimicrobial resistance in Helicobacter pylori therapy - the impact of omeprazole. Gut 1997;41(suppl 1):A89.

26 Bell GD, Bate CM, Axon AT, et al. Addition of metronidazole to omeprazole/amoxycillin dual therapy increases the rate of Helicobacter pylori eradication: a double-blind, randomized trial. Aliment Pharmacol Ther 1995;9:513-20.

27 Veldhuyzen van Zanten S, Hunt RH, Cockeram A, et al. Adding once-daily omeprazole $20 \mathrm{mg}$ to metronidazole/ amoxicillin treatment for Helicobacter pylori gastritis: a randomized, double-blind trial showing the importance of
metronidazole resistance. Am f Gastroenterol 1998;93:5-10.

28 Lind T, Bardhan KD, Bayerdörffer E, et al. The MACH 2 study: optimal Helicobacter pylori therapy needs omeprazole and can be assessed by UBT [abstract]. Gastroenterology 1997;112:A200.

29 Tompkins DS, Perkin J, Smith C. Failed treatment of Helicobacter pylori infection associated with resistance to clarithromycin. Helicobacter 1997;2:185-7.

30 Miyaji H, Azuma T, Ito S, et al. Susceptibility of Helicobacter pylori isolates to metronidazole, clarithromycin and amoxycillin in vitro and in clinical treatment in Japan. Aliment Pharmacol Ther 1997;11:1131-6.

31 Marais A, Monteiro L, Lamouliatte $\mathrm{H}$, et al. Cag negative status of Helicobacter pylori is a risk factor for failure of PPI-based triple therapies in non-ulcer dyspepsia [abstract]. Gastroenterology 1998;114:A214. 
32 Huang JQ, Hunt RH. Are one-week anti-H. pylori treatments more effective in patients with peptic ulcer disease (PUD) than in those with non-ulcer dyspepsia (NUD)? A meta-analysis [abstract]. Am f Gastroentero 1998;93:119.

33 Go MF, Graham DY. Presence of the cagA gene in the majority of Helicobacter pylori strains is independent of whether the individual has duodenal ulcer or asymptomatic gastritis. Helicobacter 1996;1:107-11.

34 Catalano F, Catanzaro R, Bronga A, et al. Duodenal ulcer and functional dyspepsia: different susceptibility of Helicobacter pylori to eradicating therapy [abstract]. Gastroenterology 1997;112:A84.

35 Luna P, del Castillo G, Farías R, et al. Efficacy and tolerability of a seven-day triple scheme with pantoprazole, amoxicillin and clarithromycin for eradication of Helicobacter pylori [abstract]. Gastroenterology 1997;112:A202.

36 Moayyedi P, Langworthy H, Shanahan K, et al. Comparison of one or two weeks of lansoprazole, amoxicillin, and clarithromycin in the treatment of Helicobacter pylori. thromycin in the treat

37 Bazzoli F, Zagari RM, Fossi S, et al. Short-term low-dose triple therapy for the eradication of Helicobacter pylori. Eur f Gastroenterol Hepatol 1994;6:773-7.

38 Huang JQ, Wilkinson JM, Hunt RH. Lansoprazole (L) given bid is better than once daily in combination with antibiotic(s) for eradicating $\mathrm{H}$. pylori infection: a metaanalysis [abstract]. Am f Gastroenterol 1997;92:1616.

39 Laine L, Estrada R, Trujillo M, et al. Randomized comparison of differing periods of twice-a-day triple therapy for the eradication of Helicobacter pylori. Aliment Pharmacol The 1996;10:1029-33.
40 Ricci C, Menegatti M, Landi F, et al. Failure of eradicating Helicobacter pylori (HP) treatment: which is the next step? Digestion 1998;59(suppl 3):427.

41 Houben MHMG, van de Beek D, van 't Hoff BWM, et al. PPI-triple therapy failure in Helicobacter pylori $(\mathrm{Hp})$ infection: re-treatment with PPI-triple therapy or quadruple therapy? Digestion 1998;59(suppl 3):413

42 Sheu BS, Wu JJ, Yang SB, et al. One-week proton pump inhibitor-based triple therapy is effective to eradicate the residual $\mathrm{H}$. pylori after dual therapy. Digestion 1998; 59(suppl 3): 430

43 Rinaldi V, Zullo A, Pugliano F, et al. The management of failed dual or triple therapy for Helicobacter pylori eradication. Aliment Pharmacol Ther 1997;11:929-33.

44 Gisbert JP, Boixeda D, Bermejo F, et al. Re-treatment after H. pylori eradication failure: which therapy is better? Digestion 1998;59(suppl 3):410.

45 vd Wouden EJ, Thijs JC, van Zwet AA, et al. Metronidazole resistance does not influence the efficacy of triple therapy with ranitidine bismuth citrate (RBC), clarithromycin (CLA) and metronidazole (MET) for H. pylori (Hp) infection [abstract]. Gastroenterology 1998;114:A323.

46 Graham DY, Hoffman J, Osato MS, et al. New therapy for metronidazole or clarithromycin resistant Helicobacter pylori: FOC or FOM [abstract]. Gastroenterology 1998;114: A138.

47 Moshkowitz M, Konikoff FM, Peled Y, et al. One week triple therapy with omeprazole, clarithromycin and tinidazole or Helicobacter pylori: differing efficacy in previously treated and untreated patients. Aliment Pharmacol Ther 1996;10:1015-19. 